\title{
Chromospheric magnetic reconnection caused by photospheric flux emergence: implications for jet-like events formation
}

\author{
J. Y. Ding ${ }^{1,2}$, M. S. Madjarska ${ }^{1}$, J. G. Doyle ${ }^{1}$, and Q. M. Lu$^{2}$ \\ 1 Armagh Observatory, College Hill, Armagh BT61 9DG, N. Ireland \\ e-mail: jyd@arm.ac.uk \\ 2 School of Earth and Space Sciences, University of Science and Technology of China, Hefei 230026, PR China \\ Received 10 August 2009 / Accepted 17 December 2009
}

\section{ABSTRACT}

\begin{abstract}
Magnetic reconnection in the low atmosphere, e.g. chromosphere, is investigated in various physical environments. Its implications for the origination of explosive events (small-scale jets) are discussed. A 2.5-dimensional resistive magnetohydrodynamic (MHD) model in Cartesian coordinates is used. It is found that the temperature and velocity of the outflow jets as a result of magnetic reconnection are strongly dependent on the physical environments, e.g. the magnitude of the magnetic field strength and the plasma density. If the magnetic field strength is weak and the density is high, the temperature of the jets is very low $\left(\sim 10^{4} \mathrm{~K}\right)$ as well as its velocity $\left(\sim 40 \mathrm{~km} \mathrm{~s}^{-1}\right)$. However, if environments with stronger magnetic field strength $(40 \mathrm{G})$ and smaller density (electron density $N_{\mathrm{e}}=2 \times 10^{10} \mathrm{~cm}^{-3}$ ) are considered, the outflow jets reach higher temperatures of up to $6 \times 10^{5} \mathrm{~K}$ and a line-of-sight velocity of up to $130 \mathrm{~km} \mathrm{~s}^{-1}$ which is comparable with the observational values of jet-like events.
\end{abstract}

Key words. magnetohydrodynamics (MHD) - Sun: chromosphere - Sun: transition region - Sun: UV radiation - magnetic fields

\section{Introduction}

Jet-like events, first reported by Brueckner \& Bartoe (1983), are characterised by non-Gaussian spectral line profiles. Dere et al. (1991) suggested that they are produced by bi-directional jets as a result of magnetic reconnection. To date, these jetlike events (often called explosive events) are mainly observed in spectral lines formed at transition region temperatures (Dere 1994; Chae et al. 1998; Innes et al. 2001; Madjarska \& Doyle 2003), although observations of explosive events in chromospheric lines are also reported. For example, Madjarska \& Doyle (2002) presented the temporal evolution of different temperature plasma using high cadence (10 s) observations obtained with the solar ultraviolet measurement of emitted radiation (SUMER) spectrometer, and found a time delay in the response of the $\mathrm{S}$ VI $933 \AA\left(2 \times 10^{5} \mathrm{~K}\right)$ line with respect to Ly $6\left(2 \times 10^{4} \mathrm{~K}\right)$, with the Ly 6 line responding earlier. They concluded that the jet-like events may first appear at chromospheric temperatures. In follow-up work, Doyle et al. (2005) reported on a joint SUMER, coronal diagnostic spectrometer (CDS) on board the Solar Heliospheric Observatory and TRACE imager study, confirming the possibility that some jet-like events originate in the chromosphere. They further suggested that jet-like events could be divided into two types: one formed in the chromosphere and the other formed in the transition region. Some of the observed features are the result of spicules and/or macrospicules (Madjarska \& Doyle 2003; Madjarska et al. 2006), while others are the result of high velocity flows in small loops (Teriaca et al. 2004). In more recent work, Madjarska et al. (2009) presented observational data relating explosive events to a surge and demonstrated that the division of small-scale transient events into a number of different subgroups, for instance explosive events, blinkers, spicules, surges or just brightenings, is ambiguous, implying that the definition of a feature based only on either spectroscopic or imaging characteristics as well as insufficient spectral and spatial resolution can be incomplete.

Several numerical models were developed to study jet-like events. Sarro et al. (1999) used a 1D magnetic flux-tube model to simulate the temporal evolution of UV emission line profiles, e.g. C IV $1548.2 \AA$, in response to energy perturbations located below the transition-region. The maximum blue-shifts they obtained reach values of the order of $100 \mathrm{~km} \mathrm{~s}^{-1}$. Innes \& Tóth (1999) presented a 2D MHD study on jet-like events with different initial conditions, representative of different regions in which the reconnection occurs, e.g. the corona and chromosphere. Their conclusion was that high-velocity components in the profiles of lines formed around $10^{5} \mathrm{~K}$ can be obtained in both cases, irrespective of the initial conditions. However, heat conduction was not included, and no brightening was found at the zero velocity position of the spectral line. In their model, the initial equilibrium state consists of two regions of oppositely directed magnetic field lines, with a narrow current sheet between the two regions. Magnetic reconnection at the current sheet is initiated by introducing localized anomalous resistivity. Roussev et al. (2001a) carried out 2D MHD simulations, where jet-like events are formed during the process of magnetic reconnection. In their model, the initial magnetic field is parallel to the $y$-axis (vertical), and there is a thin current concentration formed along the $y$-axis. Magnetic reconnection is initiated by a localized increase of the magnetic diffusivity in the current concentration. Blue-shifts of the order of $100 \mathrm{~km} \mathrm{~s}^{-1}$ were obtained. By using the same model, they further extended the work and performed simulations under different physical conditions (Roussev et al. 2001b,c).

Yokoyama \& Shibata $(1995,1996)$ performed 2D magnetic reconnection to study coronal X-ray jets using both oblique and 
vertical initial coronal magnetic fields. The temperature of the hot X-ray jets they obtained reach 3 times the coronal temperature. Moreno-Insertis et al. (2008) considered magnetic reconnection triggered by flux emergence from below the photosphere using a 3D MHD model. Very strong X-ray jets with high temperature $\left(3 \times 10^{7} \mathrm{~K}\right.$ at the reconnection site $)$ and high velocity (peak velocity $400 \mathrm{~km} \mathrm{~s}^{-1}$ ) were produced. In their model, the flux emergence is very strong (maximum field strength $3.8 \mathrm{kG}$ ), which plays a very important role in forming such strong jets. Isobe et al. (2008) focused on the process of magnetic flux emergence, and presented simulations of magnetic flux emergence driven by the upward convective motion. They found that smallscale horizontal magnetic fields could be produced even when the initial magnetic field is uniform and vertical. The horizontal magnetic fields emerging from the convection zone into the photosphere undergoes magnetic reconnection with the background vertical field, which is a source of high-frequency MHD waves that may contribute to coronal heating or solar wind acceleration.

Murray et al. (2009) presented another simulation of magnetic flux emergence, where the long-term evolution of magnetic reconnection was initiated by flux emergence. A series of reconnection reversals (or oscillatory reconnection) was reported. All the flux emergence studies mentioned above did not include heat conduction and radiative effects. The latter will reduce both the temperature and the velocity of the outflow jets.

Litvinenko \& Chae (2009) discussed magnetic reconnection at different heights in the solar atmosphere, and found that the temperature and speed of the outflow jets vary by several orders. Their study was based on an extended Sweet-Parker model (Parker 1957, 1963), assuming that the inflowing magnetic energy is completely converted in the current sheet into the thermal and kinetic energies of the outflowing plasma.

In the present study, we use a 2.5-dimensional resistive MHD model in Cartesian coordinates to investigate magnetic reconnection in the low atmosphere, e.g. chromosphere. We discuss its implications for jet-like events. Here, we use various physical environments representing the lower atmosphere with different magnetic field strengths and densities. In our model, the magnetic reconnection is triggered by newly emerging magnetic fluxes from below the photosphere which reconnect with the preexisting background magnetic field lines in the chromosphere.

The physical model is described in Sect. 2, while the numerical results are shown in Sect. 3. No background heating is included in this model, the implications of which are discussed in Sect. 4.

\section{Physical model and numerical methods}

\subsection{Basic equations}

For 2.5-dimensional MHD studies in Cartesian coordinates $(x, y, z)$, one may introduce a magnetic flux function $\psi(t, x, y)$ to express the magnetic field by

$\boldsymbol{B}=\nabla \times(\psi \hat{z})+B_{z} \hat{z}$.

The 2.5-D resistive MHD equations are in the following nondimensional form

$$
\begin{aligned}
& \frac{\partial \rho}{\partial t}+\nabla \cdot(\rho \boldsymbol{v})=0, \\
& \frac{\partial \boldsymbol{v}}{\partial t}+\boldsymbol{v} \cdot \nabla \boldsymbol{v}+\frac{1}{\rho} \nabla p-\frac{1}{\rho} \boldsymbol{j} \times \boldsymbol{B}-\boldsymbol{g}=0, \\
& \frac{\partial \psi}{\partial t}+\boldsymbol{v} \cdot \nabla \psi-\eta \Delta \psi=0
\end{aligned}
$$

$$
\begin{aligned}
& \frac{\partial B_{z}}{\partial t}+\boldsymbol{v} \cdot \nabla B_{z}+B_{z} \nabla \cdot \boldsymbol{v}-\boldsymbol{B} \cdot \nabla v_{z}-\nabla \cdot\left(\eta \nabla B_{z}\right)=0 \\
& \frac{\partial T}{\partial t}+\boldsymbol{v} \cdot \nabla T+(\gamma-1) T \nabla \cdot \boldsymbol{v}-\frac{2(\gamma-1) \eta}{\rho \beta_{0}} \boldsymbol{j} \cdot \boldsymbol{j}-\frac{C_{1}}{\rho} Q+\frac{C_{2}}{\rho} L_{\mathrm{r}}=0
\end{aligned}
$$

where $\rho$ is the mass density, $\boldsymbol{v}$ is the flow velocity, $T$ is the temperature, $p=\rho T$ is the gas pressure, $\boldsymbol{g}$ is the gravitational acceleration, $\gamma(=5 / 3)$ is the adiabatic index, $\eta$ is the dimensionless magnetic diffusivity which is inverse to the magnetic Reynolds number, $\beta_{0}$ is the characteristic ratio of the gas pressure to the magnetic pressure, $\boldsymbol{j}=\nabla \times \boldsymbol{B}$ is the electric current density, $Q=\nabla \cdot\left[T^{5 / 2}(\boldsymbol{B} \cdot \nabla T) \boldsymbol{B} / B^{2}\right]$ is the field-aligned heat conduction function, and $L_{\mathrm{r}}$ is the radiative losses. Here we assume that the plasma is fully ionized, therefore, the dimensionless form of $L_{\mathrm{r}}$ is explicitly given by

$L_{\mathrm{r}}=\rho^{2} \Lambda(T)$,

where $\Lambda(T)$ is the radiative loss function, of which the expression used is that given by Rosner et al. (1978). Various physical environments representing the solar atmosphere with different characteristic values are examined. The different cases can be divided into two groups according to the characteristic value of $\beta_{0}$. The two groups are denoted by Exp. A and Exp. B, corresponding to $\beta_{0}=0.33$ and 0.033 , respectively. For both groups, the following characteristic values are kept invariant and taken as basic units: $\rho_{0}=3.33 \times 10^{-10} \mathrm{~kg} \mathrm{~m}^{-3}$ for the mass density, $T_{0}=10^{4} \mathrm{~K}$ for the temperature, $L_{0}=500 \mathrm{~km}$ for the length, and $v_{0}=\sqrt{R T_{0}}=12.8 \mathrm{~km} \mathrm{~s}^{-1}$ for the velocity, where $R$ is the gas constant. In terms of the expression $\beta_{0}=2 \mu \rho_{0} R T_{0} / B_{0}^{2}$ where $\mu$ is the vacuum magnetic permeability, we can derive the characteristic values of the magnetic field strength $B_{0}=6 \mathrm{G}$ for Exp. A and $20 \mathrm{G}$ for Exp. B. The dimensionless coefficient of the heat conduction and radiative losses are given by

$C_{1}=(\gamma-1) \kappa_{0} T_{0}^{7 / 2} /\left(\rho_{0} L_{0} v_{0}^{3}\right)$ and $C_{2}=(\gamma-1) L_{0} \rho_{0} /\left(v_{0}^{3} m_{\mathrm{p}}^{2}\right)$,

respectively, where $\kappa_{0}=10^{-11} \mathrm{~W} \mathrm{~m}^{-1} \mathrm{~K}^{-7 / 2}$ is the classical conductivity coefficient, and $m_{\mathrm{p}}$ is the proton mass. In the present study the $x$ - and $z$-axes are in the horizontal plane, and $y$-axis represents the height of the solar atmosphere. Therefore, the dimensionless form of the gravitational acceleration is expressed as

$\boldsymbol{g}=-g \hat{y}$,

where $g=0.271 \mathrm{~km} \mathrm{~s}^{-2} L_{0} / v_{0}^{2}$.

\subsection{Initial state}

The initial magnetic field is a linear force-free field taken to be in the following form

$\psi=\left\{\begin{array}{c}((4 \omega / \pi) \cos (\pi x / 2 \omega)-4 \omega / \pi) \sqrt{2 \mu \rho_{0} R T_{0} / \beta_{0}}, \\ \text { if }|x|<\omega \\ (-2|x|-4 \omega / \pi+2 \omega) \sqrt{2 \mu \rho_{0} R T_{0} / \beta_{0}}, \\ \text { if }|x| \geq \omega,\end{array}\right.$

$B_{z}= \begin{cases}2 \cos (\pi x / 2 \omega) \sqrt{2 \mu \rho_{0} R T_{0} / \beta_{0}}, & \text { if }|x|<\omega \\ 0, & \text { if }|x| \geq \omega\end{cases}$

where $\omega$ is the half width of the force-free region (current sheet) and is set to 0.1 . The unit of magnetic field strength is taken as $B_{0}=\sqrt{2 \mu \rho_{0} R T_{0} / \beta_{0}}$. The lower $\beta_{0}$ is, the stronger the magnetic field strength. In order to represent the solar atmosphere from 
J. Y. Ding et al.: Chromospheric magnetic reconnection caused by photospheric flux emergence

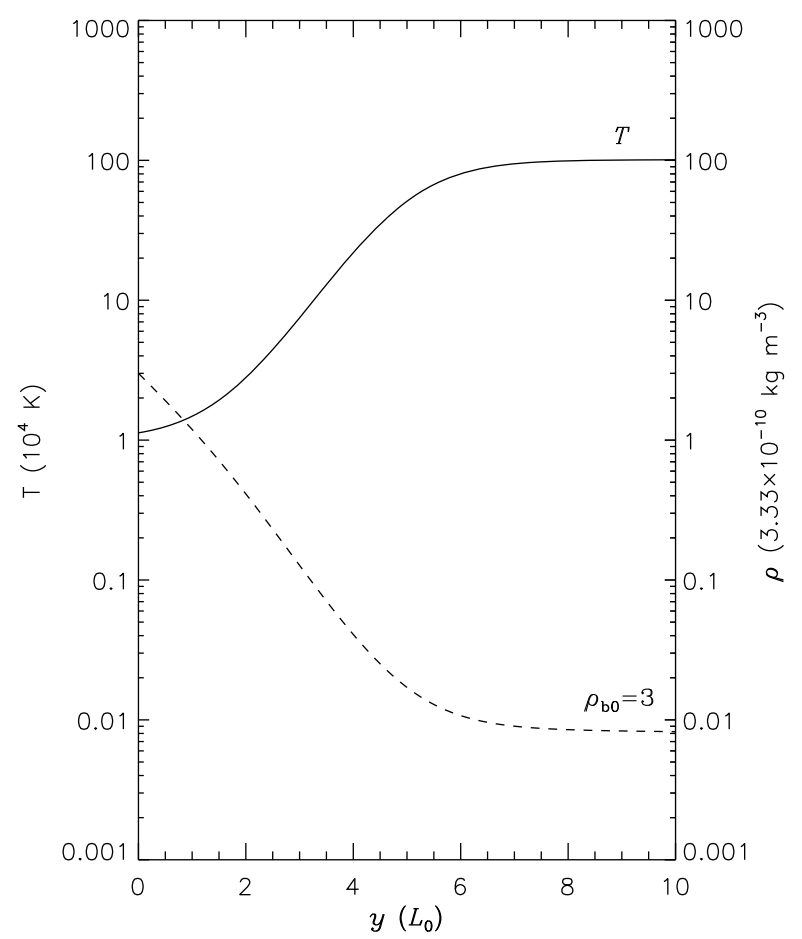

Fig. 1. The distribution of the initial temperature $(T$, solid line) and mass density ( $\rho$, dashed line) with height $(y)$. The density distribution corresponds to the case where $\rho_{\mathrm{b} 0}=3$.

the chromosphere to the upper transition region, the profile of the initial temperature $T$ is given by

$T(0, x, y)=T_{\text {in }}=1+\Delta T\left\{1+\tanh \left[\epsilon\left(y-y_{s}\right)\right]\right\}$

where $2 \triangle T=100$ defines the relative temperature jump across the transition region, $\epsilon=0.6$ describes the steepness of the temperature profile and $y_{s}=5$ is the position of the middle transition region. The temperature is equal to 1 at the bottom and is 101 at the top. Then, the mass density is calculated from the hydrostatic equilibrium equation, $\nabla p=-\rho g \hat{y}$. In order to obtain a unique solution for mass density, we specify the density value at the bottom as a boundary condition. It is easy to deduce that both the temperature and the mass density of the initial state are dependent only on the $y$-coordinate, and are uniform along the $x$ coordinate. Therefore, the initial mass density at the bottom can be expressed by one parameter, $\rho_{\mathrm{b} 0}$. Figure 1 shows one example for the initial distribution of the temperature and the density along the $y$-coordinate when $\rho_{\mathrm{b} 0}=3$.

In this study, two initial states with different values of $\rho_{\mathrm{b} 0}$ are considered for each of Exp. A and B. In total, four cases which represent four physical environments are discussed here, all of which are listed below:

- Exp. A1: $\beta_{0}=0.33, \rho_{\mathrm{b} 0}=3$ (weak magnetic field and high mass density);

- Exp. A2: $\beta_{0}=0.33, \rho_{\mathrm{b} 0}=0.1$ (weak magnetic field and low mass density);

- Exp. B1: $\beta_{0}=0.033, \rho_{\mathrm{b} 0}=3$ (strong magnetic field and high mass density);

- Exp. B2: $\beta_{0}=0.033, \rho_{\mathrm{b} 0}=0.1$ (strong magnetic field and low mass density).

In all the cases, the dimensionless form of the initial magnetic field is the same, and the change of the magnetic field strength is only due to the change of $\beta_{0}$. Moreover, the dimensionless form of the magnetic flux emergence is the same in all the cases, as well as the dimensionless form of the magnetic diffusivity, which will be discussed in the following section.

We should note that most numerical studies consider an artificial hearting source, however we do not consider it here. The main reason is that we do not know where that artificial heating term comes from, thus, in our model, the initial solar background atmosphere is not in thermal equilibrium. In order to reduce the influence of the flows that are driven by the re-distribution of the heat and radiative cooling from the initial background atmosphere, the calculations in our paper are carried out over a shorter interval (about $80 \mathrm{~s}$ ), compared to the time scale for heat conduction and cooling from the initial configuration.

\subsection{New magnetic flux emergence}

Here, we do not discuss the specific physical mechanism for the flux emergence which is beyond the scope of this paper, although recent observations with the Hinode Solar Optical Telescope (SOT; Tsuneta et al. 2008) reveal that the emergence of granularscale magnetic flux is ubiquitous on the solar surface (Centeno et al. 2007; Ishikawa et al. 2008; Isobe et al. 2008; Okamoto et al. 2008). Instead, what we are interested in is the physical response of the chromosphere after the magnetic flux has emerged from below the photosphere and reached the upper atmosphere, the chromosphere and transition region. The emergence of new magnetic flux is implemented numerically by changing the boundary conditions of the magnetic field at the bottom, which is described below in detail. First, we specify the flux emergence region that is taken to be $1 \leq x \leq 4$. Then, we change the magnetic flux function at $y=0$ in the emergence region to a new value, $\psi_{\mathrm{e}}$, according to a function of $x$ and time, expressed by

$\psi_{\mathrm{e}}=\psi_{\text {in }}(x)+\alpha\left|\left(\psi_{\text {in }}(x)-\psi_{\text {in }}(1)\right) \times\left(\psi_{\text {in }}(x)-\psi_{\text {in }}(4)\right)\right| t / t_{\mathrm{e}}$,

$1 \leq x \leq 4,0 \leq t \leq t_{\mathrm{e}}$,

where $\psi_{\text {in }}$ is the initial magnetic flux function at the base, $t_{\mathrm{e}}$ is the duration for the flux emergence, and $\alpha$ controls the magnitude and orientation of the emerging flux. When $t$ is greater than $t_{\mathrm{e}}$, the newly specified flux distribution at the base remains invariant in the emergence region. The flux distribution outside the emergence region is fixed to be the same as that of the initial field, see Eq. (8). In all numerical examples discussed below, $t_{\mathrm{e}}$ is taken to be $80 \mathrm{~s}$ and $\alpha$ is -1.2 .

As new flux emerges, a current sheet will be formed at the interface between the newly emerging and pre-existing magnetic fluxes. Then, anomalous resistivity is introduced to initiate magnetic reconnection. The distribution of the resistivity is localised, and taken to be of the following form

$\eta= \begin{cases}\eta_{\mathrm{m}} \min \left(1, j / j_{\mathrm{c}}\right), & \text { if }|x| \geq 1, y \leq 3, \\ 0, & \text { elsewhere, }\end{cases}$

where $\eta_{\mathrm{m}}=0.002$, and $j_{\mathrm{c}}=10$, is the critical value of the current density $(j)$. No magnetic reconnection is considered at the current sheet of the initial background magnetic field within $|x|<\omega$, see Eq. (11).

There are thus two current sheets in our model. One is at the interface between the newly emerging magnetic flux and the pre-existing background magnetic flux and the other is along $y$ at around $x=0$ which belongs to the background magnetic field. This is because we only want to study the magnetic reconnection 
(a) $t=0.0 \mathrm{~s}$

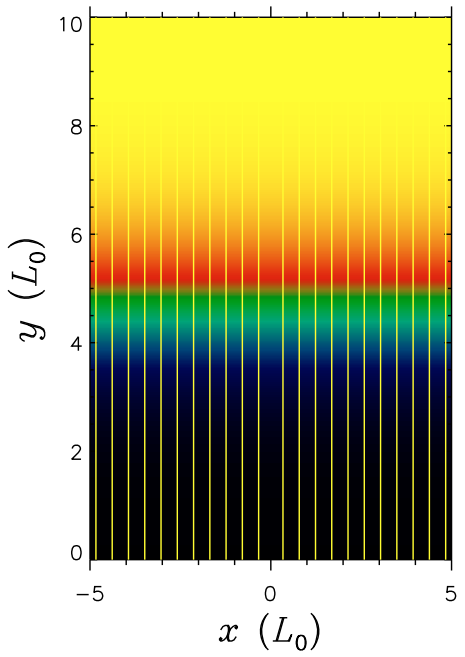

(b) $t=32.0 \mathrm{~s}$

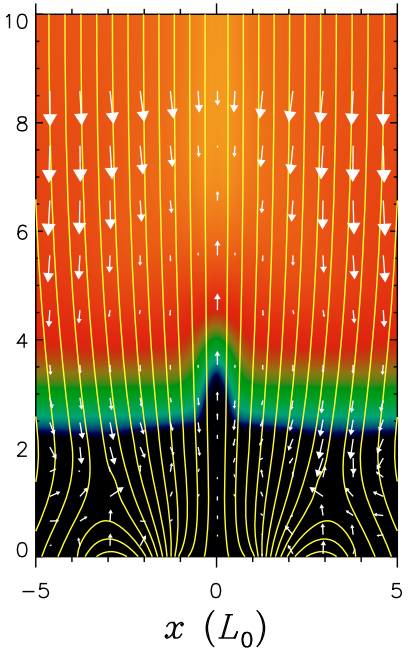

(c) $t=64.0 \mathrm{~s}$

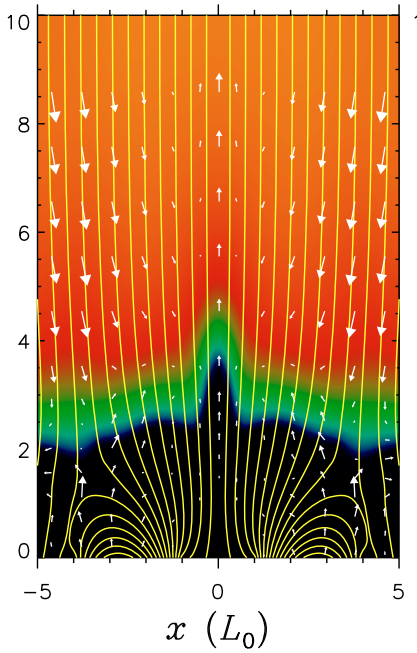

(d) $t=80.0 \mathrm{~s}$

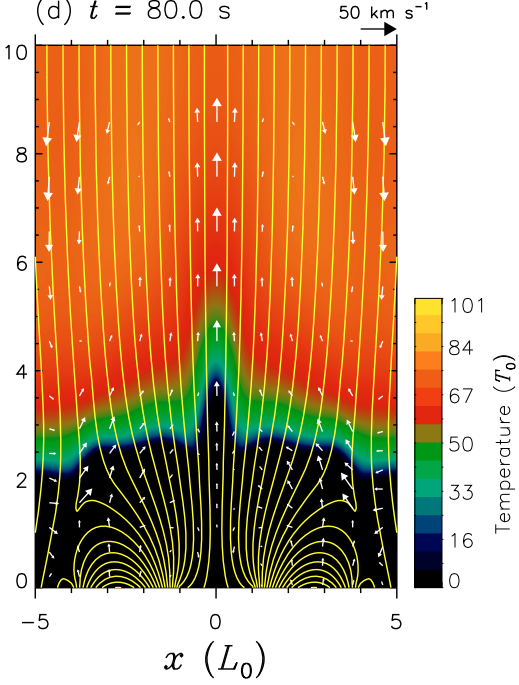

Fig. 2. The evolution of the magnetic field (solid line), temperature (colour) and velocity (arrows), corresponding to Exp. A1, where $\beta_{0}=$ 0.33 and $\rho_{\mathrm{b} 0}=3$.

occurring on the former one. Here, we introduce a localized resistivity (at $|x|>1, y<3$ ). The current is almost 0 outside this region $(|x|>1, y<3)$, except for that around $x=0$.

The initial state used here contains a current sheet around $x=0$, which is not the same as the one used by Yohokyama \& Shibata $(1995,1996)$ (where the initial magnetic field is a uniform vertical one). The reason for using non-uniform magnetic field is that it is easier for us to compare the results in this paper with those where reconnections at both current sheets are considered. The two current sheets represent different magnetic topology: one is formed between open vertical field lines while the other is between open and closed ones. The reconnection between the newly emerging and pre-existing magnetic fluxes will have influence on the reconnection between the open vertical fluxes and vice versa. In this case, the polarity of the emerging flux will be an important factor. However, in this paper, based on the fact that no magnetic diffusion is considered at the initial current sheet around $x=0$, the influence of the initial current sheet on the results can be regarded as the influence of different left-boundary conditions if compared with the uniform vertical field case. As the reconnection occurs on the right-hand side of the emerging flux arcade, far away from the initial current sheet around $x=0$, there is no significant differences in the results if a uniform initial field is used instead.

\subsection{Computational domain}

The dimensionless size of the computational domain is $-5 \leq$ $x \leq 5$ and $0 \leq y \leq 10$. Because of symmetry, we only implement calculation in the right half region $(0 \leq x \leq 5,0 \leq y \leq 10)$. As for the boundary conditions, we fix all quantities at the base, and treat the top $(y=10)$ and the right-hand side $(x=5)$ as open boundaries. Furthermore, we use symmetrical conditions for the left boundary $(x=0)$. A multistep implicit scheme (Hu 1989) is used to solve Eqs. (2)-(6).

We adopt $399 \times 400$ grid points, which is based on the consideration of the balance between resolution and computation time. We can also adopt more grid points so as to get better resolution, but the computation time will become very long then, especially for the case in which strong magnetic field strength and low mass density are presented. Uniform meshes are adopted in both the $x$ - and $y$-directions.

\section{Numerical results}

We present here magnetic reconnection which occurs in the low atmosphere and is triggered by newly emerging magnetic flux. Four cases representing different physical environments are discussed. According to the strength of the magnetic field, the results are divided into two groups: Exp. A and B. For each group, two initial states with different mass density at the bottom, $\rho_{\mathrm{b} 0}$, are considered.

\section{1. $\operatorname{Exp} . A\left(\beta_{0}=0.33\right)$}

First, let us discuss the case where $\rho_{\mathrm{b} 0}=3$ (i.e. electron density $N_{\mathrm{e}}=6 \times 10^{11} \mathrm{~cm}^{-3}$ ), which means the mass density at the bottom is large, corresponding to Exp. A1. The results are shown in Fig. 2, where the evolution of the magnetic field, temperature and velocity are displayed. At the beginning of the experiment, downward flows are seen, especially in the middle temperature region (see Fig. 2b), which is caused by heat conduction and radiative cooling. As the new flux emerges, an electric current sheet is formed at the right interface between the newly emerging and pre-existing magnetic fluxes. Then magnetic reconnection occurs at the current sheet producing bi-directional outflows: upward and downward. The outflow jet in each direction will be accelerated by magnetic tension forces and shocks along the boundary of the jet (Petschek 1964), and reaches its maximum velocity $\left(v_{\text {jet }}\right)$ somewhere in front of the jet. For example, at $t=32 \mathrm{~s}$, the upward flow jet reaches its maximum velocity, $v_{\text {jet }}=40 \mathrm{~km} \mathrm{~s}^{-1}$, at $x=3.2, y=1.1$, whereas the diffusion region is located at a lower altitude, around $x=4.0, y=0.3$. As the magnetic reconnection continues, the upward flow jet propagating towards higher altitudes will interact with the downward flow of the background field, forming a shock at the interface between them. The shock will impede the downward flow, so that the downward flow first becomes weaker and then even reverses its direction, see Fig. 2. During the process of magnetic reconnection, both the magnitude and orientation of $v_{\text {jet }}$ change dynamically. But, no matter how $v_{\text {jet }}$ changes, it is far smaller than that of the jet-like events observed by SUMER. In spite of this, it is comparable with the characteristic local Alfvén speed around the diffusion region, as predicted by the fast magnetic reconnection model (Petschek 1964; Priest \& Forbes 1986). In the 

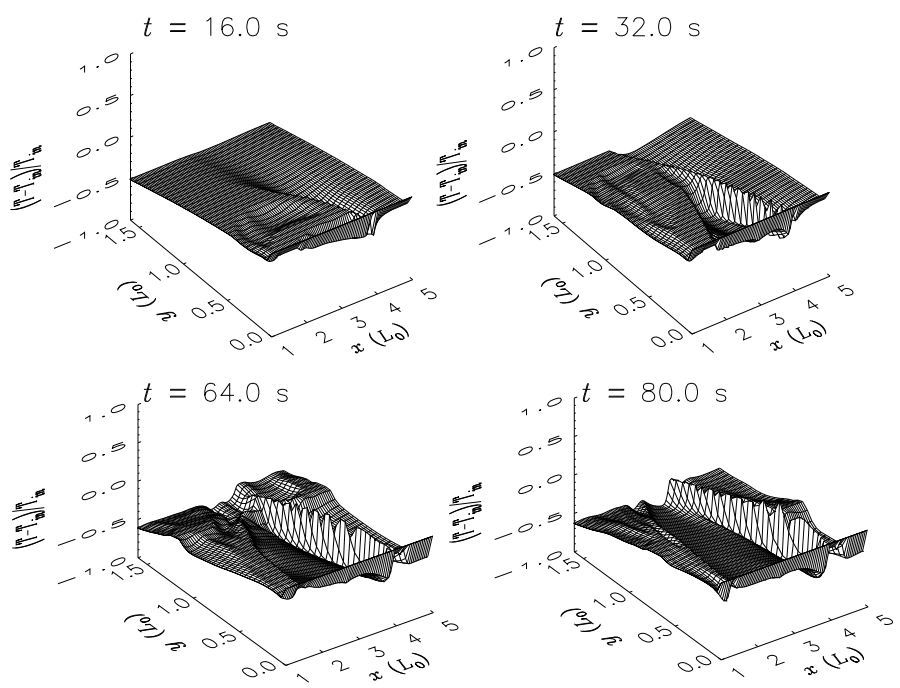

Fig. 3. Relative changes in the temperature, $\left(T-T_{\text {in }}\right) / T_{\text {in }}$ for Exp. A1, with respect to the initial temperature $\left(T_{\mathrm{in}}\right)$, are shown at four times in the region $1 \leq x \leq 5,0 \leq y \leq 1.6$.

following sections, the velocity $v_{\text {jet }}$ we give is the maximum one during the time from $0-80 \mathrm{~s}$. Also, the velocity in the line-ofsight relative to $v_{\text {jet }}$ will be presented. In this case, the downward flow jet (as a result of magnetic reconnection) can not be clearly shown, because the $\mathrm{X}$-point is very low in our model. Its height is 0.2 above the bottom at $t=32 \mathrm{~s}$, and is 0.8 at $t=80 \mathrm{~s}$. The height is so small that only a small part of the downward flow jet is displayed in our computation domain. Moreover, as mentioned above, the outflow jet will reach its maximum velocity somewhere in front of the diffusion region, which means the maximum velocity of the downflow jet may be located below the bottom of our computational domain. The downward flow jet that can be displayed in our model reaches $12 \mathrm{~km} \mathrm{~s}^{-1}$ at $t=80 \mathrm{~s}$. In the following discussions, we will focus on the upflow jet.

Figure 2 is drawn in such a large scale that the temperature evolution around the magnetic reconnection region has a very poor resolution. In order to better illustrate the temperature evolution in this region, we plot only a small part $(1 \leq$ $x \leq 5,0 \leq y \leq 1.6$ ) of the computation domain in Fig. 3 (and in successive plots), where the relative changes in the temperature, $\left(T-T_{\text {in }}\right) / T_{\text {in }}$, with respect to the initial temperature, $T_{\text {in }}$, are shown. The relative changes in mass density, $\left(\rho-\rho_{\text {in }}\right) / \rho_{\text {in }}$, with respect to the initial density, $\rho_{\text {in }}$, are shown in Fig. 4 at the same times and in the same region as in Fig. 3.

Peak structures are clearly seen on each rim of the flux emergence region for both the relative changes in densities and temperatures. As the magnetic reconnection occurs, the plasma in the diffusion region is heated, and the hot plasma is pulled out by the magnetic tension force in either direction along the current concentration, resulting in the peak structure on the right-hand side of the flux emergence region in both Figs. 3 and 4, compared with those in the inflow region. Along the peak structure, the temperature reaches its maximum in the diffusion region, while the density is minimum in the diffusion region. After the magnetic reconnection, part of the reconnected magnetic field lines extend outward towards $x=0$ (see Fig. 2), resulting in a negative horizontal velocity component of the upflow jet. Accordingly, on the left-hand side of the flux emergence region, plasma accumulates and a strong shock is created which causes the formation of the another peak structure in the relative changes in density plot; the peak structure in the relative changes in temperature
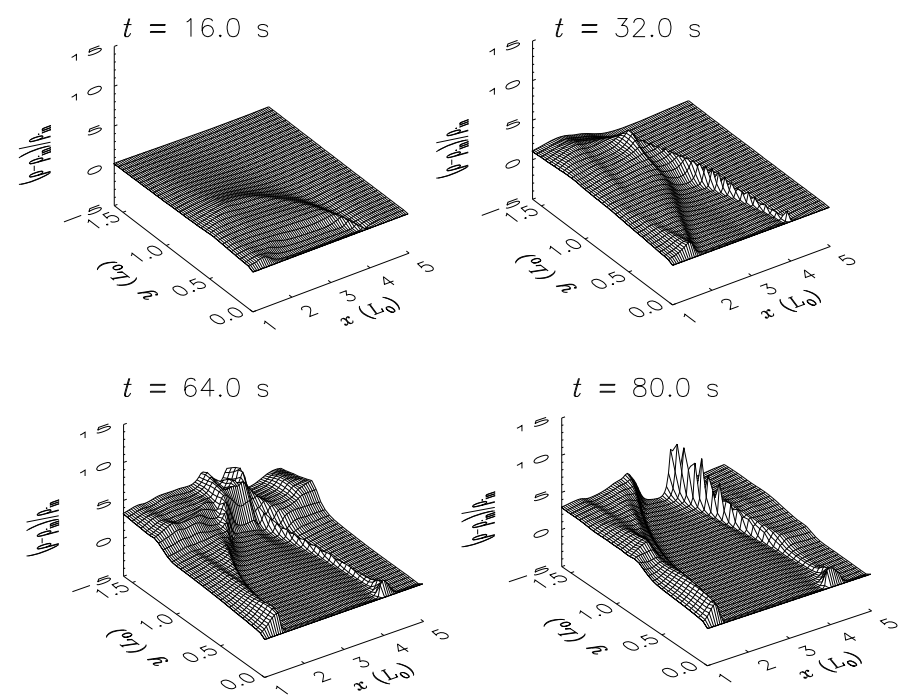

Fig. 4. Relative changes in mass density, $\left(\rho-\rho_{\text {in }}\right) / \rho_{\text {in }}$ for Exp. A1, with respect to the initial density $\left(\rho_{\text {in }}\right)$, are shown at the same times and in the same region as shown in Fig. 3.

plot is due to the heating effect of the shock. In the following, the temperatures at two locations are analysed: one in the diffusion region and the other at the location of $v_{\text {jet }}$. They are denoted by $T_{\max }$ and $T_{\text {jet }}$, respectively.

Even though the plasma temperature along the current concentration is heated through magnetic reconnection, the relative changes in temperature are negative all over the region shown in Fig. 3. Both the temperatures $T_{\max }$ and $T_{\text {jet }}$ are very low, around $10^{4} \mathrm{~K}$. In the following, we make quantitative analysis of how this happens.

From Eqs. (6) and (7), we get

$\frac{\partial T}{\partial t} \sim \frac{2(\gamma-1) \eta}{\rho \beta_{0}} j^{2}-C_{2} \rho \Lambda(T)$

where the contribution of the Joule dissipation and radiative losses are considered. For simplicity, the first term on the righthand side of Eq. (12) is denoted by $E_{1}$, and the second term by $E_{2}$. Because both the temperature and its gradient are very small at low temperature regions, the heat conduction term $\left(\sim 10^{-5}\right)$ is neglected here. Note that the Joule dissipation and radiative losses play opposite roles in the evolution of temperature, one heats the plasma and the other cools it. From Eq. (12), we find that $E_{1}$ is inversely proportional to the density, whereas $E_{2}$ is proportional to the density. In the diffusion region where the density is small, $E_{1}$ is $\sim 80$, and $E_{2}$ is $\sim 50$. The net effect is that the plasma in the diffusion region is heated. However, the heated plasma is pulled out of the diffusion region and the cold plasma outside the diffusion region is pushed in, so that the plasma in the diffusion region cools. Outside the diffusion region, the plasma density along the current sheet increases by several times with respect to the initial density (see Fig. 4), so that $E_{2}$ increases to $\sim 200$ at maximum, resulting in a fast temperature decrease. If the plasma temperature is below $10^{4} \mathrm{~K}$, radiative losses are reduced to zero, so that the relative changes in the plasma temperature is flat along the outflow region (located along the "high" temperature ridge on the right-hand side of the flux emergence region seen in the bottom right panel of Fig. 3). Furthermore, outside the diffusion region, the outflow plasma will expand, which will cool the plasma. The contribution of the plasma expansion to plasma cooling is $(\gamma-1) T \nabla \cdot v \sim 40$. In the diffusion region, the plasma expansion will also cool the plasma there, however its 

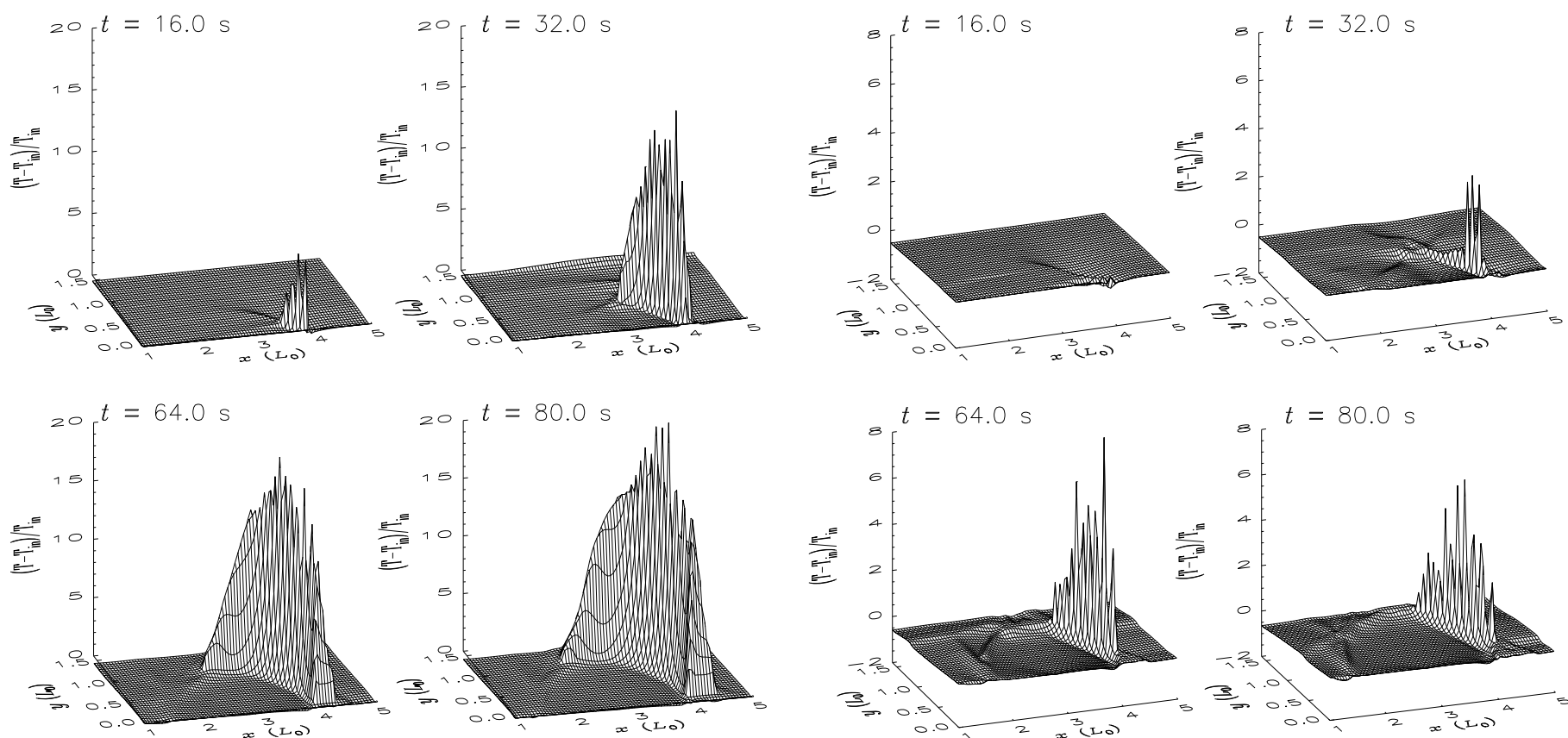

Fig. 5. Relative changes in the temperature, $\left(T-T_{\text {in }}\right) / T_{\text {in }}$ for Exp. A2, with respect to the initial temperature $\left(T_{\text {in }}\right)$, are shown at four times in the region $1 \leq x \leq 5,0 \leq y \leq 1.6$.

contribution is very small, only about 4 . A large plasma cooling region is found around $x=2-4$ almost reaching $y=2$ (see Fig. 3), located in the inflow region. This is because of the plasma expansion in the inflow region, of which the contribution to plasma cooling reaches 40 . Even though the contributions of plasma expansion to plasma cooling are of almost the same order in the inflow region and the outflow region, the plasma temperature decreases more strongly in the former than in the latter. This is caused by the fact that there is no hot plasma flowing into the inflow region, whereas hot plasma heated in the diffusion region is expelled out into the outflow region.

It seems that small-scale jets can not originate in a physical environment like Exp. A1 where high mass density and weak magnetic strength are presented, as both the velocity and the temperature of the outflow jets are very low. What will be the result if the physical environment with the same magnetic strength but a lower mass density is considered? The analysis shows that, if the density is lower, more plasma can be heated (because both the radiative losses and the ratio of the radiative losses to Joule dissipation is proportional to $\rho^{2}$ ).

Figure 5 shows the evolution of the temperature for the case of Exp. A2 where $\beta_{0}=0.33$ and $\rho_{\mathrm{b} 0}=0.1$ (this corresponds to magnetic field strength $B_{0}=6 \mathrm{G}$, electron density $N_{\mathrm{e}}=2 \times 10^{10} \mathrm{~cm}^{-3}$ ). Significant relative changes in temperature are clearly seen in this case and the plasma in the diffusion region is heated to the temperature of $T_{\max }=2.8 \times 10^{5} \mathrm{~K}$. The maximum velocity of the outflow jet caused by magnetic reconnection and its temperature are also analysed for Exp. A2. We find that $v_{\text {jet }}$ reaches up to $85 \mathrm{~km} \mathrm{~s}^{-1}$ and $T_{\text {jet }}$ is about $2 \times 10^{5} \mathrm{~K}$. Both are larger than that obtained in Exp. A1. The direction of $v_{\text {jet }}$ is $32^{\circ}$ with respect to the line of sight.

\section{2. $\operatorname{Exp} . B\left(\beta_{0}=0.033\right)$}

In this section, the magnetic reconnection takes place in a physical environment with a strong magnetic field. Figure 6 shows

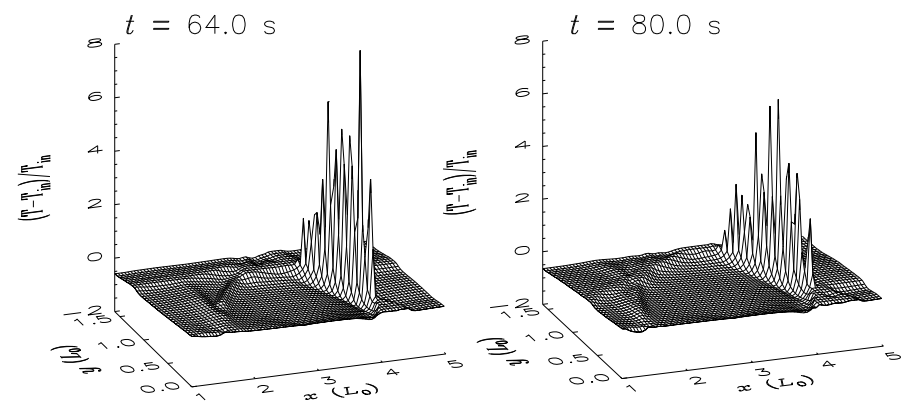

Fig. 6. Relative changes in the temperature, $\left(T-T_{\text {in }}\right) / T_{\text {in }}$ for Exp. B1, with respect to the initial temperature $\left(T_{\text {in }}\right)$, are shown at four times in the region $1 \leq x \leq 5,0 \leq y \leq 1.6$.
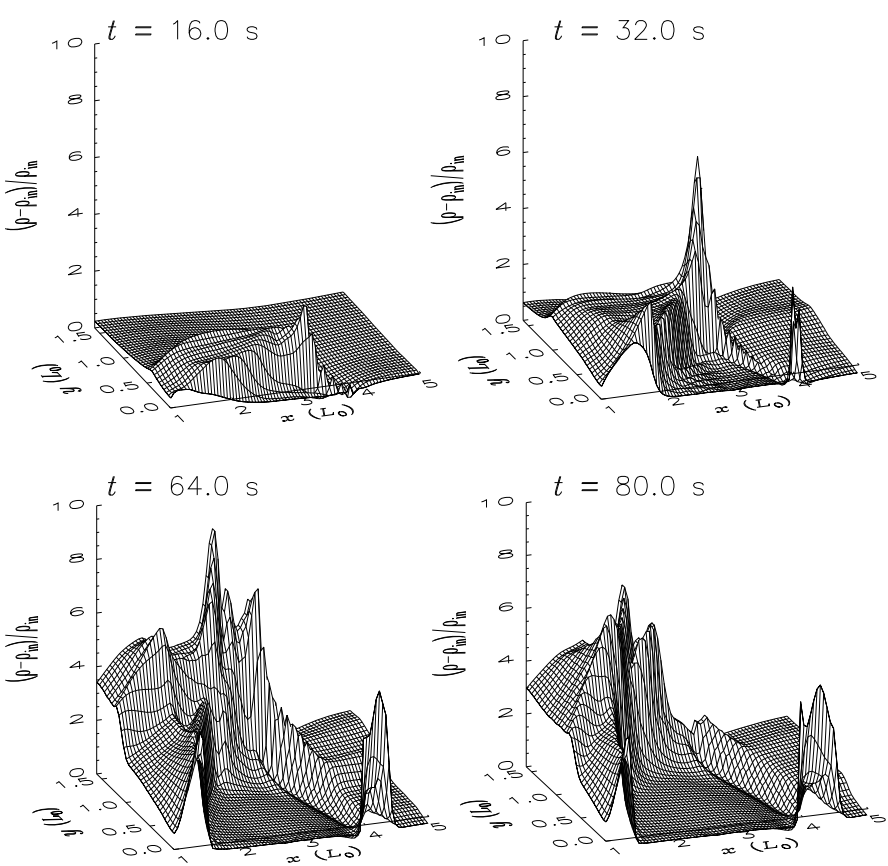

Fig. 7. Relative changes in mass density, $\left(\rho-\rho_{\text {in }}\right) / \rho_{\text {in }}$ for Exp. B1, with respect to the initial density $\left(\rho_{\text {in }}\right)$, are shown at the same times and in the same region as shown in Fig. 6.

the evolution of the temperatures for the case of Exp. B1 where $\beta_{0}=0.033$ and $\rho_{\mathrm{b} 0}=3$ which corresponds to $B_{0}=20 \mathrm{G}$ and $N_{\mathrm{e}}=6 \times 10^{11} \mathrm{~cm}^{-3}$. This represents a physical environment with a strong magnetic field and a high mass density. Figure 7 shows the relative changes in density. Peak structures are present in the relative changes in both the temperature and density on each rim of the flux emergence region. In the diffusion region, $T_{\max }$ is $1.5 \times 10^{5} \mathrm{~K}$. For the upflow jet, $v_{\text {jet }}$ reaches $60 \mathrm{~km} \mathrm{~s}^{-1}$, directed at $21^{\circ}$ with respect to the line of sight, and $T_{\text {jet }}$ is about $8 \times 10^{4} \mathrm{~K}$. Comparing the results with those in Exp. A1 where we use the 

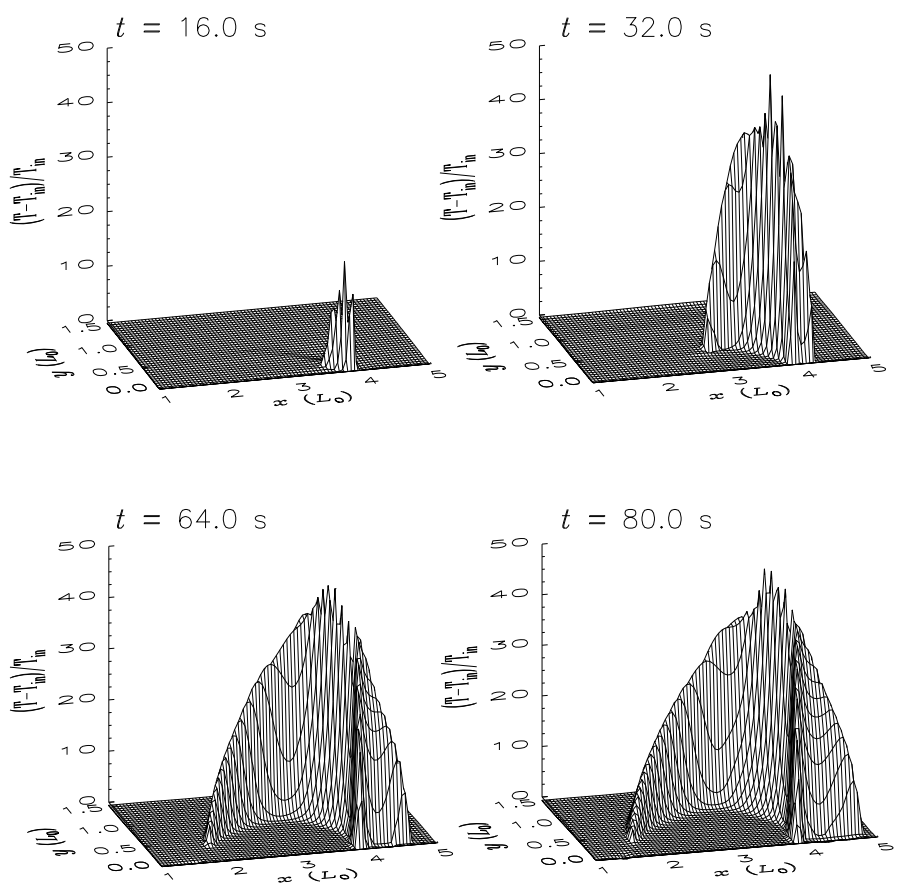

Fig. 8. Relative changes in the temperature, $\left(T-T_{\text {in }}\right) / T_{\text {in }}$ for Exp. B 2 , with respect to the initial temperature $\left(T_{\text {in }}\right)$, are shown at four times in the region $1 \leq x \leq 5,0 \leq y \leq 1.6$.

Table 1. Summary of the results for both Exp. A and B. $v_{y}$ denotes the line-of-sight component of $v_{\text {jet }}$.

\begin{tabular}{ccccc}
\hline \hline Quantity & Exp. A1 & Exp. A2 & Exp. B1 & Exp. B2 \\
\hline$T_{\max }(\mathrm{K})$ & $\sim 1 \times 10^{4}$ & $2.8 \times 10^{5}$ & $1.5 \times 10^{5}$ & $6.4 \times 10^{5}$ \\
$T_{\text {jet }}(\mathrm{K})$ & $\sim 1 \times 10^{4}$ & $2 \times 10^{5}$ & $8 \times 10^{4}$ & $6 \times 10^{5}$ \\
$v_{\text {jet }}\left(\mathrm{km} \mathrm{s}^{-1}\right)$ & 40 & 85 & 60 & 150 \\
$v_{y}\left(\mathrm{~km} \mathrm{~s}^{-1}\right)$ & 32 & 72 & 56 & 130 \\
\hline
\end{tabular}

same mass density at the bottom but with a weaker magnetic field strength, we find that the plasma is heated to higher temperature for the case of Exp. B1 compared to Exp. A1. This is because Joule dissipation becomes stronger as the magnetic field strength increases. In the case of Exp. B1, $E_{1} \sim 800$, in the diffusion region, whereas $E_{2} \sim 500$. Even though $E_{2}$ increases as a result of the increase of $\Lambda(T)$, the net effect is that more energy is available to heat the plasma in the diffusion region. The relative changes in the temperature are manifested as a maximum in the diffusion region and decrease monotonously in either direction along the current concentration. The maximum velocity of the outflow jets is larger in Exp. B1 than in Exp. A1. This is because as the ratio of the magnetic pressure to gas pressure increases, it leads to higher acceleration of the outflow plasma as the magnetic field strength increases.

Figure 8 shows the results for the case of Exp. B2, where $\beta_{0}=0.033$ and $\rho_{\mathrm{b} 0}=0.1$ (i.e. $B_{0}=20 \mathrm{G}$ and $N_{\mathrm{e}}=2 \times$ $10^{10} \mathrm{~cm}^{-3}$ ). In this case, the plasma in the diffusion region is heated to $T_{\max }=6.4 \times 10^{5} \mathrm{~K}$. The maximum velocity of the outflow jets, $v_{\text {jet }}$, reaches up to $150 \mathrm{~km} \mathrm{~s}^{-1}$, and is $130 \mathrm{~km} \mathrm{~s}^{-1}$ in the line-of-sight. The temperature at the location of $v_{\text {jet }}$ is $6 \times 10^{5} \mathrm{~K}$. Note that, for the case with low mass density, the heat conduction term will become more and more important as an energy loss in the diffusion region compared to the radiative loss term, when the temperature of the plasma increases to a higher value. All the results for both Exp. A and B are summarized in Table 1.

\section{Discussions}

In the present paper, we investigate magnetic reconnection in the solar chromosphere, looking for an answer to whether jetlike events can originate at lower temperatures. We consider magnetic reconnection triggered by newly emerging magnetic flux. Several physical environments representing the chromosphere with different magnetic field strengths and densities are discussed. Our results quantitatively show that the temperatures and velocities of the bi-directional jets caused by magnetic reconnection are strongly dependent on the physical conditions of the atmosphere, such as magnetic field strength and mass density. In the case of where the magnetic field strength is high (Exp. B2) and the density is low, we obtain the highest temperatures and the largest velocities among all our simulations. The plasma in the diffusion region is heated up to $6.4 \times 10^{5} \mathrm{~K}$, and the maximum velocity of the outflow jets reaches $150 \mathrm{~km} \mathrm{~s}^{-1}$. However, in the case of a low magnetic field strength (Exp. A1) and high density, the temperature and the velocities of the jets are the lowest of all the cases. The temperature is about $10^{4} \mathrm{~K}$ and the maximum velocity of the jets is only $40 \mathrm{~km} \mathrm{~s}^{-1}$. Either an increase in the magnetic field strength or a decrease of the density will raise the ratio of the Joule dissipation to the radiative losses. That will heighten the heating effect compared to cooling and will lead to a high increase in the temperature of the plasma in the diffusion region. That will also raise the ratio of the magnetic pressure to the gas pressure, resulting in enhanced acceleration of the plasma ejected outward from the diffusion region (which will lead to the appearance of high velocity jets).

For the two cases, Exp. A and B, the amounts of the emerging flux are not the same, which implies that the stronger the magnetic field strength the larger the amount of newly emerging magnetic flux. We can also obtain outflows with high velocity and high temperature even if a smaller amount of new flux, e.g. the same as that in case A, is emerged in case B. Moreover, in case B where a larger amount of new flux emerges, the temperature and velocity of the outflow jets reach their maximum at about $t=40 \mathrm{~s}$, not at the end of the flux emergence process. Therefore, the difference in the amount of emerging flux between case $\mathrm{A}$ and $\mathrm{B}$ is not very important in determining different results in the two cases presented. Instead, the magnetic field strength of the initial background magnetic field and the initial density structure plays a more significant role. If we do all the experiments with constant flux emergence, the amount of the newly emerging flux will be very small in case B, compared with that of the background field. So that the height of the emerging arcade is lower in case B than in case A at any given time, with the result that reconnection occurs at different heights in the two cases.

The flow velocities derived here can be compared with Roussev et al. $(2001 \mathrm{~b}, \mathrm{c})$ who found blue-shifts of the order of $100 \mathrm{~km} \mathrm{~s}^{-1}$ under the physical environment of electron density $3 \times 10^{10} \mathrm{~cm}^{-3}$ and magnetic field $8 \mathrm{G}$. These authors also found that the choice of initial state and the consideration of non-equilibrium ionization was crucial to their modelling. Even though various initial physical environments were explored in their work, the plasma $\beta$ on the current sheet was the same in all the cases. Both the plasma $\beta$ and the thermal energy were assumed to be uniform in the $y$-direction with the reconnection happening in the transition region. The maximum jet velocities they obtained under different physical environments did differ at the begining of the experiments, however, they approached to almost the same value as time went on (see top-left panel in Fig. 9 in Roussev et al. 2001b). In our model, the maximum jet 
velocities are different for all the cases. The highest velocity jets are obtained under the environment with almost the same density as in Roussev et al. (2001b), but stronger magnetic field (20 G). When the reconnection occurs in the chromosphere, high velocity jets-like features are possible if a larger field strength is used. Besides, we do not consider transient ionization.

In the flux emergence studies of Yokoyama \& Shibata (1995, 1996) and Moreno-Insertis et al. (2008), reconnection occurs at coronal hights where the plasma temperature is high and the electron density is low $\left(10^{10} \mathrm{~cm}^{-3}\right)$, even though new flux emerges from beneath the photosphere. Therefore, it is easier to obtain jets with high velocities and high temperatures. In the Isobe et al. (2008) model, magnetic reconnection between newly formed horizontial fields and background vertical fields took place at chromospheric heights. The chromospheric electron density is much higher $\left(>10^{12} \mathrm{~cm}^{-3}\right)$ than that in our model, so that the reconnection jets velocity they obtained is very slow, about $30 \mathrm{~km} \mathrm{~s}^{-1}$. In the Murray et al. (2009) model, oscillatory reconnection is driven by the global imbalance of the forces between the neighbouring flux systems. At each subsequent reconnection reversal, the system is closer to equilibrium. Once an equilibrium state is reached, all reconnection ceases, therefore, the maximum temperature and maximum velocity $\left(50 \mathrm{~km} \mathrm{~s}^{-1}\right)$ achieved in the outflow jets decreases in each subsequent reconnection phase. All the flux emergence studies mentioned above did not include heat conduction and radiative effects. The latter will reduce both the temperature and the velocity of the outflow jets.

In comparison to Litvinenko \& Chae (2009) model where radiative losses were assumed to be of less importance, they become significantly important in our model when the density and temperature are large. Their study was based on the assumption that the inflowing magnetic energy is completely converted into thermal and kinetic energies of the outflowing plasma. Therefore, the temperature and speed of the outflow jets are larger in their model than those in ours at the same height for the same parameter.

By using our model we would like to give a possible explanation as to why no brightening is obtained at zero velocity of the emission spectral lines in the study by Innes \& Tóth (1999). In their study, the magnetic field strength and mass density in the chromosphere was about $6 \mathrm{G}$ and $2.8 \times 10^{-10} \mathrm{~kg} \mathrm{~m}^{-3}$, respectively, which corresponds to $\beta_{0}=1.3$ and $\rho_{\mathrm{b} 0}=0.8$ in our model. Our calculations show that in this case the maximum temperature in the diffusion region is less than $5 \times 10^{4} \mathrm{~K}$ and therefore, no brightening could appear at the zero velocity position of the spectral line.

The radiative loss model that we use at the base is the opticalthin approximation. McClymont \& Canfield (1983) considered optical-depth effects and proposed an optical-thick model for the radiative loss function. By comparing the two models, we find that the optical-depth effects become important primarily for the plasma at temperatures below $3 \times 10^{4} \mathrm{~K}$, where the contribution of hydgron is dominant. This means, in our model, that the radiative cooling effect is over-evaluated for the cool plasma at temperatures below $3 \times 10^{4} \mathrm{~K}$. The temperature of the cool plasma should be raised if we take into account the optical-depth effects in our model. However, even if we consider optical-depth effects, the corrected temperature of the cool plasma will not exceed $3 \times 10^{4} \mathrm{~K}$. Otherwise, radiative loss will become too strong and the plasma will start to cool again. Considering that the jet-like events discussed here correspond to hot jets at transition-region temperatures, optical depth effects will have less influnce.
As mentioned in Sect. 2, background heating is not considered in our model, therefore the initial state is not in thermalequilibrium. We take the case of Exp A1, shown in Fig. 2, as an example to examine the change of the background. If no emergence takes place, the plasma temperature at the top boundary will decrease by $27 \%$ by the end of $80 \mathrm{~s}$. Because of background cooling, downward flow will occur, which reaches about $40 \mathrm{~km} \mathrm{~s}^{-1}$ at maximum during $0-80 \mathrm{~s}$. The high velocity region is mainly located at high altitude (transition region or above), whereas the downward flow around the diffusion region is slower (less than $10 \mathrm{~km} \mathrm{~s}^{-1}$ ).

Priest \& Forbes (1986) studied mechanisms of magnetic reconnection analytically. They introduced a new parameter $(b)$ and produced a series of analytical solutions. They found that the type of reconnection regime and the rate of reconnection depends sensitively on the parameter $b$ which characterises the inflow conditions. The Petschek $(b=0)$ solution is just one particular member, corresponding to a weak fast-model plasma expansion. However, in our model, there is a fast plasma expansion across the inflow region, and the diffusion region lengthens as reconnection occurs. Therefore, the reconnection event here resembles more the fast-mode expansion solutions with $b>0$ in Priest \& Forbes (1986).

In this study, reconnection occurs on the right-hand side of the emerging flux arcade. If reconnection occurs on the lefthand side of the emerging flux arcade, e.g. the polarity of the emerging flux changes, the magnetic field strength on the lefthand side will decrease faster as reconnection goes on. This is because symmetric boundary conditions are used on the left, whereas open boundary is used on the right. As magnetic field strength decreases, magnetic reconnection will become weaker. Despite of this, there will not be significant differences in the results if reconnection occurs on the left-hand side of the emerging flux arcade (because the jets reach their maximum velocity faster than the decrease of the magnetic field strength). The magnetic field strength of the initial field on the left-hand side of the flux emergence region is still very strong, when the jets velocity reach their maximum (for instance, at around $40 \mathrm{~s}$ in case B). If investigating long-term evolution, a larger computational domain, compared with the flux emergence region, is necessary to weaken the influence of boundary conditions.

In the present paper, we mainly concentrate on the hot component of the outflow jets caused by magnetic reconnection, and obtain jets with a high temperature and a high velocity under certain circumstances. We also produce cool jets in the temperature range of $(2 \pm 0.2) \times 10^{4} \mathrm{~K}$. Taking the case of Exp. B2 as an example. The line-of-sight velocity of the cool upflow jets reaches $25 \mathrm{~km} \mathrm{~s}^{-1}$. This shows one possibility that some cool jets, e.g. spicules, and hot jets may originate from the same phenomenon, chromospheric magnetic reconnection, and they may just depict different parts and/or different stages of the same event. In the case of weak magnetic field strength but high mass density, the cool jets disappear, which shows that the origination of spicules may also have some correlations with the physical environment. We relegate further study of the relationship between spicules, jet-like events, and magnetic reconnection to future work.

Acknowledgements. Research at Armagh Observatory is grant-aided by the N. Ireland Dept. of Culture, Arts and Leisure (DCAL). This work was supported via grant ST/F001843/1 from the UK Science and Technology Facilities Council. M.M. and J.G.D. thank the International Space Science Institute, Bern for the support of the team "Small-scale transient phenomena and their contribution to coronal heating". 
J. Y. Ding et al.: Chromospheric magnetic reconnection caused by photospheric flux emergence

\section{References}

Brueckner, G. E., \& Bartoe, J.-D. F. 1983, ApJ, 272, 329

Centeno, R., Socas-Navarro, H., Lites, B., et al. 2007, ApJ, 666, L137

Chae, J., Wang, H., Lee, C.-Y., Goode, P. R., \& Schuhle, U. 1998, ApJ, 497, L109

Dere, K. P. 1994, Adv. Space Res., 14, 13

Dere, K. P., Bartoe, J.-D. F., Brueckner, G. E., Ewing, J., \& Lund, P. 1991, J. Geophys. Res., 96, 9399

Doyle, J. G., Ishak, B., Ugarte-Urra, I., Bryans, P., \& Summers, H. P. 2005, A\&A, 439, 1183

Hu, Y. Q. 1989, J. Comput. Phys., 84, 441

Innes, D. E. 2001, A\&A, 378, 1067

Innes, D. E., \& Toth, G. 1999, Sol. Phys., 185, 127

Ishikawa, R., Tsuneta, S., Ichimoto, K., et al. 2008, A\&A, 481, L25

Isobe, H., Proctor, M. R. E., \& Weiss, N. O. 2008, ApJ, 679, L57

Litvinenko, Y. E., \& Chae, J. 2009, A\&A, 495, 953

Madjarska, M. S., \& Doyle, J. G. 2002, A\&A, 382, 319

Madjarska, M. S., \& Doyle, J. G. 2003, A\&A, 403, 731

Madjarska, M. S., Doyle, J. G., Hochedez, J.-F., \& Theissen, A. 2006, A\&A, 452, L11
Madjarska, M. S., Doyle J. G., \& de Pontieu, B. 2009, ApJ, 701, 253

McClymont, A. N., \& Canfield, R. C. 1983, ApJ, 265, 497

Moreno-Insertis, F., Galsgaard, K., \& Ugarte-Urra, I. 2008, ApJ, 673, L211

Murray, M. J., van Driel-Gesztelyi, L., \& Baker, D. 2009, A\&A, 494, 329

Okamoto, T. J., Tsuneta, S., Lites, B. W., et al. 2008, ApJ, 673, L215

Parker, E. N. 1957, J. Geophys. Res., 62, 509

Parker, E. N. 1963, ApJS, 8, 177

Petschek, H. E. 1964, in AAS-NASA Symposium on the Physics of Solar Flares, ed. W. N. Hess, NASA Spec. Publ., SP-50, 425

Priest, E. R., \& Forbes, T. G. 1986, J. Geophys. Res., 91, 5579

Rosner, R., Tucker, W. H., \& Vaiana, G. S. 1978, ApJ, 220, 643

Roussev, I., Galsgaard, K., Erdelyi, R., \& Doyle, J. G. 2001a, A\&A, 370, 298

Roussev, I., Galsgaard, K., Erdelyi, R., \& Doyle, J. G. 2001b, A\&A, 375, 228

Roussev, I., Doyle, J. G., Galsgaard, K., \& Erdelyi, R. 2001c, A\&A, 380, 719

Sarro, L. M., Erdelyi, R., Doyle, J. G., \& Perez, M. E. 1999, A\&A, 351, 721

Teriaca, L., Banerjee, D., Falchi, A., Doyle, J. G., \& Madjarska, M. S. 2004, A\&A, 427, 1065

Tsuneta, S., Ichimoto, K., Katsukawa, Y., et al. 2008, Sol. Phys., 249, 167

Yokoyama, T., \& Shibata, K. 1995, Nature, 375, 42

Yokoyama, T., \& Shibata, K. 1996, PASJ, 48, 353 